\title{
Hierarchical discretization of the PMCHWT formulation with jump current discontinuities for the scattering analysis of ferromagnetic objects
}

\author{
Eduard Ubeda $^{1}$ Ivan Sekulic $^{1} \quad$ Juan M. Rius ${ }^{1}$
}

\begin{abstract}
In the discretization of the Poggio-Miller-ChanHarrington-Wu-Tsai (PMCHWT) formulation by the Method of Moments (MoM), the unknown currents are usually expanded with the divergence-conforming RWG set. Recently, the discretization of the PMCHWT formulation with the monopolar-RWG basis functions, discontinuous across edges, has been successfully developed through a volumetrictetrahedral testing scheme. We present a novel even-surface odd-volumetric monopolar-RWG PMCHWT-discretization that relies on the rearrangement of the monopolar-RWG set in terms of the RWG and the odd-monopolar-RWG subsets. This scheme offers improved accuracy for a wider range of heights of the testing tetrahedral elements than the volumetrically tested monopolar-RWG PMCHWT-discretization in the analysis of small sharp-edged ferromagnetic targets.
\end{abstract}

\section{INTRODUCTION}

The integral formulation Poggio-Miller-ChanHarrington-Wu-Tsai (PMCHWT) [1][2] is widely used in the scattering analysis of penetrable targets. In the standard Method-of-Moment discretization of the PMCHWT formulation, the unknown electric and magnetic currents, defined over the surface-boundary of the object, are usually expanded with the RWG basis functions, an example of divergenceconforming set. These basis functions are especially convenient since the hypersingular Kernel contributions, arising in the expansion of scalar potentials, electric and magnetic, are cancelled out thanks to the normal continuity of the expanded currents across the edges arising from the discretization. Furthermore, these schemes are conforming with respect to the function spaces of the integral operators and therefore ensure converging solutions [3]. However, for the successful implementation of these edge-based schemes, the underlying mesh has to be conformal, i.e. every pair of adjacent facets can only intersect over a common edge. In practice, the generation of such a mesh becomes a complicated engineering task that often results in elements of disparate sizes and impedance matrices with high condition numbers. Moreover, domain decomposition schemes relying on divergence conforming basis functions require the artificial enclosure of the sub-regions involved thus introducing additional sets of unknowns [4]. These pitfalls are mitigated with the recent development of a facet-based discretization of the PMCHWT [5]. Whereas the unknown electric and magnetic currents are expanded with the monopolar-RWG set, the scattered fields are tested over small tetrahedral entities attached to the boundary surface, inside the region where, according to equivalence principle, fields must be zero [5]. The hypersingular Kernel contributions can then be evaluated numerically. Interestingly, this implementation shows better farfield accuracy than the conventional RWG discretization in the scattering analysis of single small sharp-edged targets with moderate or high dielectric contrasts [5]. The improved accuracy, though, is mainly observed for a restricted range of heights $(H)$ of the tetrahedral entities. In this paper, we propose the hierarchical rearrangement of the monopolar-RWG space of currents, electric and magnetic, into the subspaces even monopolar- $R W G$ (unnormalized RWG) and odd monopolar- $R W G$ [6]. Although the new scheme is edge-based and therefore not amenable for the analysis of nonconformal meshes, better RCS-results are observed in the scattering analysis of single small sharp-edged ferromagnetic objects for a wider $\mathrm{H}$ range when compared with the volumetrically tested PMCHWT implementation.

\section{EVEN-ODD PMCHWT-DISCRETIZATION}

The monopolar-RWG basis functions are defined as the RWG basis functions inside the triangular facets but with no normal continuity constraint across edges; hence, two independent contributions arise at both sides of edges [6]. The space spanned by the monopolar-RWG basis functions, $\left\{\boldsymbol{m}_{n}\right\}$, can be decomposed into two subspaces that are spanned by the even monopolar-RWG and the odd monopolarRWG subsets, respectively, $\left\{\boldsymbol{m}_{n}^{\mathrm{e}}\right\}$ and $\left\{\boldsymbol{m}_{n}^{\mathrm{o}}\right\}$. These are defined in terms of the two contributions $\boldsymbol{f}_{n}^{l}(\boldsymbol{r})$ and $\boldsymbol{f}_{n}^{2}(\boldsymbol{r})$ defined at the two facets $\left(S_{n}^{1}\right.$ and $\left.S_{n}^{2}\right)$ that share the $n$th edge [6] as

$$
\begin{aligned}
& \boldsymbol{m}_{n}^{\mathrm{e}}(\boldsymbol{r})=\left\{\begin{array}{ll}
\boldsymbol{f}_{n}^{l}(\boldsymbol{r}) & \boldsymbol{r} \in S_{n}^{1} \\
-\boldsymbol{f}_{n}^{2}(\boldsymbol{r}) & \boldsymbol{r} \in S_{n}^{2}
\end{array}\right\} \\
& \boldsymbol{m}_{n}^{\mathrm{o}}(\boldsymbol{r})=\left\{\begin{array}{ll}
\boldsymbol{f}_{n}^{1}(\boldsymbol{r}) & \boldsymbol{r} \in S_{n}^{1} \\
\boldsymbol{f}_{n}^{2}(\boldsymbol{r}) & \boldsymbol{r} \in S_{n}^{2}
\end{array}\right\}
\end{aligned}
$$

${ }^{1}$ CommSensLab, Dpt. Teoria del Senyal i Comunicacions, Universitat Politècnica de Catalunya - BarcelonaTech (Spain), Email: ubeda@tsc.upc.edu 
where $1 \leq n \leq N_{e}$ and $N_{e}$ denotes the number of edges arising from the discretization of the boundarysurface. We call the subset $\left\{\boldsymbol{m}_{n}^{\mathrm{e}}\right\}$ even-monopolarRWG because it enforces normal continuity of the currents across edges. This subset corresponds with the RWG set. In light of (2), the odd-monopolarRWG subset $\left\{\boldsymbol{m}_{n}^{\circ}\right\}$ enforces the normal component of the currents at both sides of the common edge to have the same absolute value but opposite sign [6].

The electric and magnetic currents, $\boldsymbol{J}_{i}$ and $\boldsymbol{M}_{i}$, residing on each side $(i=1,2)$ of the boundary interface, are expanded in terms of the even and odd monopolar-RWG components as follows

$$
\begin{gathered}
\boldsymbol{J}_{i} \simeq \sum_{n=1}^{2 N_{e}} J_{n}^{i} \boldsymbol{m}_{n}=\sum_{n=1}^{N_{e}} a_{n}^{\mathrm{e}, i} \boldsymbol{m}_{n}^{\mathrm{e}}+\sum_{n=1}^{N_{e}} a_{n}^{\mathrm{o}, i} \boldsymbol{m}_{n}^{\mathrm{o}} \\
\boldsymbol{M}_{i} \simeq \sum_{n=1}^{2 N_{e}} M_{n}^{i} \boldsymbol{m}_{n}=\sum_{n=1}^{N_{e}} b_{n}^{\mathrm{e}, i} \boldsymbol{m}_{n}^{\mathrm{e}}+\sum_{n=1}^{N_{e}} b_{n}^{\mathrm{o}, i} \boldsymbol{m}_{n}^{\mathrm{o}}
\end{gathered}
$$

where the sequences $\quad\left\{J_{n}^{i}\right\}=\left\{a_{n}^{\mathrm{e}, i}, a_{n}^{\mathrm{o}, i}\right\} \quad$ and $\left\{M_{n}^{i}\right\}=\left\{b_{n}^{\mathrm{e}, i}, b_{n}^{\mathrm{o}, i}\right\}$ represent, respectively, the sets of unknown coefficients associated with the expansion of the electric and magnetic currents. The PMCHWT formulation relies on the approximated scattered fields associated with two regions ( 1 and 2$)$. In this paper, the index choice $i=1$ denotes the outer freespace region, whereas $i=2$ denotes the inner ferromagnetic region. The approximated scattered fields generated by the even or odd monopolar-RWG current components are defined as

$$
\begin{aligned}
& \tilde{\boldsymbol{E}}_{i}^{s \mathrm{e} / \mathrm{o}} \simeq \sum_{n=1}^{N_{e}} \eta_{i} \boldsymbol{T}_{n}^{i \mathrm{e} / \mathrm{o}} a_{n}^{i \mathrm{e} / \mathrm{o}}-\sum_{n=1}^{N_{e}} \boldsymbol{K}_{n}^{i \mathrm{e} / \mathrm{o}} b_{n}^{i \mathrm{e} / \mathrm{o}}, \quad i=1,2 \\
& \tilde{\boldsymbol{H}}_{i}^{s e / \mathrm{o}} \simeq \sum_{n=1}^{N_{e}} \boldsymbol{K}_{n}^{i \mathrm{e} / \mathrm{o}} a_{n}^{i e / \mathrm{o}}+\sum_{n=1}^{N_{e}} \frac{1}{\eta_{i}} \boldsymbol{T}_{n}^{i \mathrm{e} / \mathrm{o}} b_{n}^{i e / \mathrm{o}}, \quad i=1,2
\end{aligned}
$$

and the integral operators in (5) and (6) yield

$$
\begin{aligned}
& \boldsymbol{K}_{n}^{i e / 0}(\boldsymbol{r})=\nabla \times \iint_{S_{n}^{\prime} \cup S_{n}^{2}} G_{i}\left(\boldsymbol{r}, \boldsymbol{r}^{\prime}\right) \boldsymbol{m}_{n}^{\mathrm{e} / \mathrm{o}}\left(\boldsymbol{r}^{\prime}\right) d S^{\prime}, \quad i=1,2 \\
& \boldsymbol{T}_{n}^{i e / o}(\boldsymbol{r})=\frac{1}{j k_{i}}\left(\begin{array}{c}
\nabla \nabla \cdot \iint_{S_{n}^{\prime} \cup S_{n}^{2}} G_{i}\left(\boldsymbol{r}, \boldsymbol{r}^{\prime}\right) \boldsymbol{m}_{n}^{\mathrm{e} / \mathrm{o}}\left(\boldsymbol{r}^{\prime}\right) d S^{\prime} \\
+k_{i}^{2} \iint_{S_{n}^{\prime} \cup S_{n}^{2}} G_{i}\left(\boldsymbol{r}, \boldsymbol{r}^{\prime}\right) \boldsymbol{m}_{n}^{\mathrm{e} / \mathrm{o}}\left(\boldsymbol{r}^{\prime}\right) d S^{\prime}
\end{array}\right), \quad i=1,2
\end{aligned}
$$

where the integral operator $\boldsymbol{K}_{n}^{i e / o}(\boldsymbol{r})$ should be interpreted in the Cauchy principal value sense and $G_{i}$ denotes the Green's function of the homogeneous medium associated with region $i$; that is, $G_{i}=e^{-j k_{i} R} /(4 \pi R)$ where $R=\left|\boldsymbol{r}-\boldsymbol{r}^{\prime}\right|$. The constants $\eta_{i}$ and $k_{i}$ stand for the wave impedance and the wave number, respectively, of the $i$-th region. In this paper, they are defined in terms of the free-space wave number, $k_{0}$, and impedance, $\eta_{0}$, as

$$
k_{1}=k_{0}, \eta_{1}=\eta_{0}, k_{2}=k_{0} \sqrt{\mu_{r}}, \quad \eta_{2}=\eta_{0} \sqrt{\mu_{r}}
$$

where $\mu_{r}$ denotes the relative permeability of the target.

The discretized PMCHWT formulation is defined at the surface of the ferromagnetic object from the subtraction of the tangential components of the approximated scattered electric and magnetic fields in (5) and (6). The sets of unknowns become $\left\{c_{n}^{\mathrm{e}}\right\}=\left\{a_{n}^{\mathrm{e}}, b_{n}^{\mathrm{e}}\right\} \quad$ and $\quad\left\{c_{n}^{\mathrm{o}}\right\}=\left\{a_{n}^{\mathrm{o}}, b_{n}^{\mathrm{o}}\right\} \quad$ because $a_{n}^{\mathrm{e} / \mathrm{o}}=a_{n}^{1 \mathrm{e} / \mathrm{o}}=-a_{n}^{2 \mathrm{e} / \mathrm{o}} \quad$ and $\quad b_{n}^{\mathrm{e} / \mathrm{o}}=b_{n}^{1 \mathrm{e} / \mathrm{o}}=-b_{n}^{2 \mathrm{e} / \mathrm{o}} \quad$ is assumed. The Galerkin testing of the even-odd monopolar-RWG discretization of the PMCHWT results in the following matrix system

$$
\begin{array}{r}
E_{p}^{i n c, \mathbf{e}}=\sum_{n=1}^{N_{e}} \mathrm{Z}_{p n}^{E, \mathbf{e}, \mathbf{e}} c_{n}^{\mathrm{e}}+\sum_{n=1}^{N_{e}} \mathrm{Z}_{p n}^{E, \mathbf{e}, \mathbf{o}} c_{n}^{\mathrm{o}} \\
H_{p}^{i n c, \mathbf{e}}=\sum_{n=1}^{N_{e}} \mathrm{Z}_{p n}^{H, \mathbf{e}, \mathbf{e}} c_{n}^{\mathrm{e}}+\sum_{n=1}^{N_{e}} \mathrm{Z}_{p n}^{H, \mathbf{e}, \mathbf{o}} c_{n}^{\mathrm{o}} \\
E_{p}^{i n c, \mathbf{0}=}=\sum_{n=1}^{N_{e}} \mathrm{Z}_{p n}^{E, \mathbf{o}, \mathrm{e}} c_{n}^{\mathrm{e}}+\sum_{n=1}^{N_{e}} \mathrm{Z}_{p n}^{E, \mathbf{o}, \mathrm{o}} c_{n}^{\mathrm{o}} \\
H_{p}^{i n c, \mathbf{0}=}=\sum_{n=1}^{N_{e}} \mathrm{Z}_{p n}^{H, \mathbf{o}, \mathrm{e}} c_{n}^{\mathrm{e}}+\sum_{n=1}^{N_{e}} \mathrm{Z}_{p n}^{H, \mathbf{o}, \mathrm{o}} c_{n}^{\mathrm{o}} \\
1 \leq p \leq N_{e}
\end{array}
$$

where the excitation vectors are defined as

$$
\begin{aligned}
E_{p}^{i n c, \mathbf{e} / \mathbf{o}} & =-\iint_{S_{p}^{1} \cup S_{p}^{2}} \boldsymbol{m}_{p}^{\mathbf{e} / \mathbf{o}} \cdot \boldsymbol{E}^{i n c} d S \\
H_{p}^{i n c, \mathbf{e} / \mathbf{o}} & =-\iint_{S_{p}^{1} \cup \cup S_{p}^{2}} \boldsymbol{m}_{p}^{\mathbf{e} / \mathbf{o}} \cdot \boldsymbol{H}^{i n c} d S
\end{aligned}
$$

and $\boldsymbol{E}^{\text {inc }}$ and $\boldsymbol{H}^{\text {inc }}$ denote the incident electric and magnetic fields. The impedance submatrices in (10) and (11) yield

$$
\mathrm{Z}_{p n}^{E, \text { e/ } /, \mathrm{e} / \mathrm{o}}=\iint_{S_{p}^{1} \cup S_{p}^{2}} \boldsymbol{m}_{p}^{\mathrm{e} / \mathrm{o}} \cdot\left(\eta_{1} \boldsymbol{T}_{n}^{1}+\eta_{2} \boldsymbol{T}_{n}^{2}-\boldsymbol{K}_{n}^{1}-\boldsymbol{K}_{n}^{2}\right)^{\mathrm{e} / \mathrm{o}} d S
$$

$$
\mathrm{Z}_{p n}^{H, \mathrm{e} / 0, \mathrm{e} / \mathrm{o}}=\iint_{S_{p}^{1} \cup S_{p}^{2}} \boldsymbol{m}_{p}^{\mathrm{e} / \mathrm{o}} \cdot\left(\boldsymbol{K}_{n}^{1}+\boldsymbol{K}_{n}^{2}+\boldsymbol{T}_{n}^{1} / \eta_{1}+\boldsymbol{T}_{n}^{2} / \eta_{2}\right)^{\mathrm{e} / \mathrm{o}} d S
$$

The odd monopolar-RWG testing integrals in (11) cannot be evaluated numerically for self or edge- 
adjacent interactions because of the hypersingular contributions of the gradient of the scalar potentials. In this work we circumvent this problem through the introduction of a non-Galerkin testing scheme. We test the fields over pairs of tetrahedral elements attached to the boundary (matching pairs of adjacent facets of the surface triangulation), inside the region where, in accordance with the equivalence theorem, fields must be null.

\subsection{Odd monopolar-SWG testing}

To reach best coupling with the odd monopolarRWG basis functions we define the odd monopolarSWG testing functions, $\left\{\boldsymbol{M}_{p}^{i \mathbf{0}}\right\}$, as [6]

$$
\boldsymbol{M}_{p}^{i \mathbf{0}}(\boldsymbol{r})=\left\{\begin{array}{ll}
\boldsymbol{v}_{p}^{i 1}(\boldsymbol{r}) & \boldsymbol{r} \in V_{p}^{i 1} \\
\boldsymbol{v}_{p}^{i 2}(\boldsymbol{r}) & \boldsymbol{r} \in V_{p}^{i 2}
\end{array}\right\}
$$

where the functions $\boldsymbol{v}_{p}^{i 1}$ and $\boldsymbol{v}_{p}^{i 2}$ denote the monopolar-SWG contributions defined over the two facet-adjacent tetrahedral elements $\left(V_{p}^{i 1}\right.$ and $\left.V_{p}^{i 2}\right)$ attached to the boundary, lying in the medium $i$ (see Fig. 1) and sharing the $p$ th edge arising from the surface triangulation [5].

We define the even-surface odd-volumetric monopolar-RWG discretization of PMCHWT from the matrix equations in (10) and (11). Whereas we keep the even surface-tested equations in (10), we replace the odd Galerkin-testing in (11) by a volumetric testing with the odd monopolar-SWG functions. The corresponding odd-volumetricallytested matrix equations become

$$
\begin{aligned}
& E_{p}^{V, i n c, \mathbf{o}}=\sum_{n=1}^{N_{e}} \mathrm{Z}_{p n}^{V, E, \mathbf{o}, \mathrm{e}} c_{n}^{\mathrm{e}}+\sum_{n=1}^{N_{e}} \mathrm{Z}_{p n}^{V, E, \mathbf{o}, \mathrm{o}} c_{n}^{\mathrm{o}} \\
& H_{p}^{V, i n c, \mathbf{o}}=\sum_{n=1}^{N_{e}} \mathrm{Z}_{p n}^{V, H, \mathbf{o}, \mathrm{e}} c_{n}^{\mathrm{e}}+\sum_{n=1}^{N_{e}} \mathrm{Z}_{p n}^{V, H, \mathbf{o}, \mathrm{o}} c_{n}^{\mathrm{o}}
\end{aligned}
$$

and the excitation vectors are now defined as

$$
\begin{aligned}
E_{p}^{V, i n c, \mathbf{0}} & =-\iint_{V_{p}^{21} \cup V_{p}^{22}} \boldsymbol{M}_{p}^{2 \mathbf{o}} \cdot \boldsymbol{E}^{i n c} d V \\
H_{p}^{V, i n c, \mathbf{0}} & =-\iint_{V_{p}^{21} \cup V_{p}^{22}} \boldsymbol{M}_{p}^{2 \mathbf{o}} \cdot \boldsymbol{H}^{i n c} d V
\end{aligned}
$$

The submatrices in (17) accordingly yield

$$
\begin{aligned}
Z_{p n}^{V, E, \mathbf{0}, \mathrm{e} / \mathrm{o}} & =\iint_{V_{p}^{21} \cup V_{p}^{22}} \boldsymbol{M}_{p}^{2 \mathbf{o}} \cdot\left(\eta_{1} \boldsymbol{T}_{n}^{1}-\boldsymbol{K}_{n}^{1}\right)^{\mathrm{e} / \mathrm{o}} d V \\
& +\iint_{V_{p}^{11} \cup V_{p}^{12}} \boldsymbol{M}_{p}^{1 \mathbf{0}} \cdot\left(\eta_{2} \boldsymbol{T}_{n}^{2}-\boldsymbol{K}_{n}^{2}\right)^{\mathrm{e} / \mathrm{o}} d V
\end{aligned}
$$

$$
\begin{aligned}
Z_{p n}^{V, H, \mathbf{0}, \mathrm{e} / \mathrm{o}} & =\iint_{V_{p}^{21} \cup V_{p}^{22}} \boldsymbol{M}_{p}^{2 \mathbf{o}} \cdot\left(\boldsymbol{K}_{n}^{1}+\frac{1}{\eta_{1}} \boldsymbol{T}_{n}^{1}\right)^{\mathrm{e} / \mathrm{o}} d V \\
& +\iint_{V_{p}^{11} \cup V_{p}^{12}} \boldsymbol{M}_{p}^{1 \mathbf{0}} \cdot\left(\boldsymbol{K}_{n}^{2}+\frac{1}{\eta_{2}} \boldsymbol{T}_{n}^{2}\right)^{\mathrm{e} / \mathrm{o}} d V
\end{aligned}
$$

In view of Fig. 1, the testing volumetric entities are attached to the boundary in the side where, for that particular region, in accordance with the equivalence theorem, fields must be zero. The accuracy of this implementation depends on the height of the testing entities $(H)$, which we characterize, in view of Fig. 1, with the same value at both regions, as a fraction of the lengths $(h)$ of the associated edges in the matching triangles.

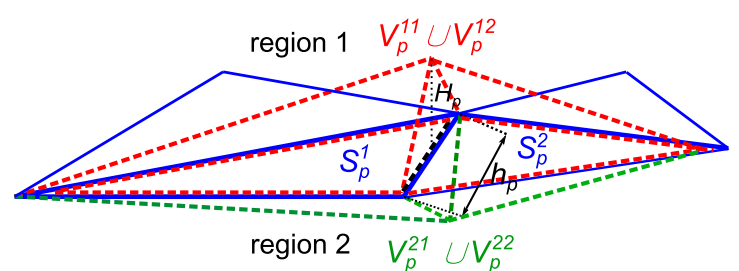

Figure 1: Volumetric testing over pairs of tetrahedral elements $V_{p}^{11} \cup V_{p}^{12}$ and $V_{p}^{21} \cup V_{p}^{22}$, attached to the pair of triangular facets $S_{p}^{1} \cup S_{p}^{2}$, and defined, respectively, inside regions 1 and 2 .

\section{NUMERICAL RESULTS}

In Fig. 2 we show bistatic RCS results near the forward direction computed with the monopolarRWG discretization of the PMCHWT with volumetric testing (PMCHWT[V-monoRWG]) [5] and the new even-surface odd-volumetric monopolarRWG discretization of PMCHWT (PMCHWT[v-eomonoRWG]) for an electrically small (side $0.1 \mathrm{~m}$ ) tetrahedron made out of cobalt $\left(\mu_{r}=250, \varepsilon_{r}=1\right)$. The height of the testing elements, $H$, is set to be $h / 100$. In Fig. 3, we show the root mean square relative $\mathrm{RCS}$-error with respect to a RCS-reference over 60 directions in the xz-plane of the PMCHWT[v-monoRWG] and PMCHWT[v-eomonoRWG] for a square pyramid made out of cobalt with side $0.071 \mathrm{~m}$. With no analytical solutions available, we compute the reference results for Figs. 2 and 3 with the conventional RWG-discretization of the PMCHWT formulation, PMCHWT[RWG], and very dense mesh (38400 unknowns for the square pyramid and 43200 unknowns for the tetrahedron). For the sake of a fair comparison, the involved implementations need to handle similar number of unknowns. Coarser triangulations are then adopted in the monopolar-RWG implementations, with two unknowns per edge, with respect to the triangulations 
of the RWG-implementations, with one unknown per edge. Therefore, in Fig. 2, for the small tetrahedron, PMCHWT[RWG] makes use of $N_{t}=784$ triangles, while PMCHWT[v-monoRWG] and PMCHWT[veo-monoRWG] adopt triangulations with $N_{t}=400$ facets. Similarly, in view of Fig. 3, for the small square pyramid, PMCHWT[RWG] makes use of $N_{t}=800$ facets while PMCHWT[v-monoRWG] and PMCHWT[v-eo-monoRWG] are computed with $N_{t}=392$ triangles. In both examples the target is impinged by an $\mathrm{x}$-polarized $+\mathrm{z}$-propagating plane wave and the free-space wavelength $\left(\lambda_{0}\right)$ is set to $1 \mathrm{~m}$.

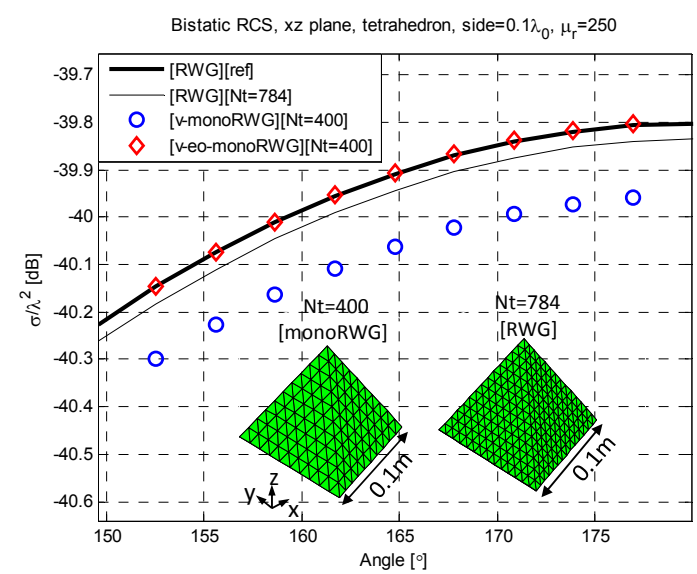

Figure 2: xz plane cut of the bistatic RCS near the forward direction computed with PMCHWT[vmonoRWG], PMCHWT[v-eo-monoRWG] and

PMCHWT[RWG] for a cobalt tetrahedron with side $0.1 \mathrm{~m}$.

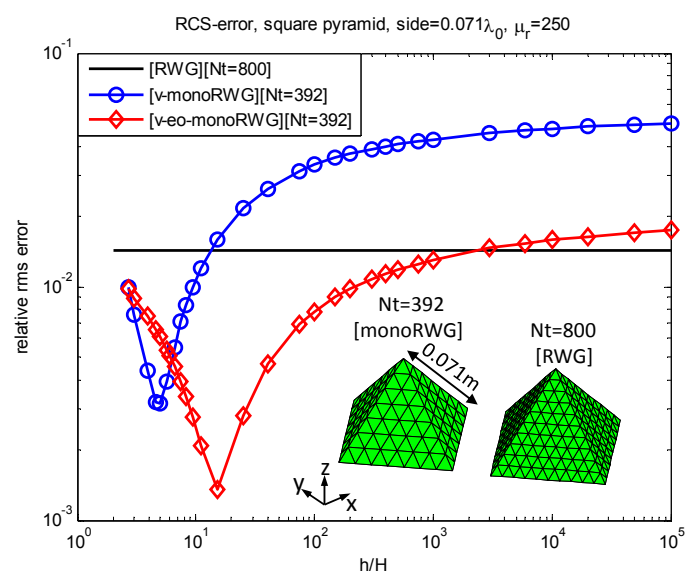

Figure 3: The relative rms RCS-errors of the monopolar-RWG PMCHWT implementations against the height of the testing entities $H$, of a cobalt square pyramid with side $0.071 \mathrm{~m}$.
From Figs. 2 and 3 we observe better far-field results for PMCHWT[v-eo-monoRWG] when compared with RCS-results computed with PMCHWT[vmonoRWG]. We attribute this improvement to the fact that the generation of the impedance matrix for PMCHWT[V-eo-monoRWG] relies partly on the well-established RWG-discretization of the PMCHWT-formulation.

\section{Acknowledgments}

This work was supported by FEDER and the Spanish Comisión Interministerial de Ciencia y Tecnología (CICYT) under projects TEC2013-47360-C3-1-P and TEC2016-78028-C3-1-P and the Unidad de Excelencia Maria de Maeztu MDM-2016-0600, which is financed by the Agencia Estatal de Investigación, Spain.

\section{References}

[1] A. J. Poggio and E. K. Miller, "Integral equation solutions of three-dimensional scattering problems," in Computer Techniques for Electromagnetics, R. Mittra, Ed. Oxford, UK: Pergamon Press, 1973, ch. 4.

[2] T. K. Wu and L. L. Tsai, "Scattering from arbitrarily-shaped lossy dielectric bodies of revolution," Radio Science, vol. 12, pp. 709-718, Sep.-Oct. 1977.

[3] R. Hiptmair and C. Schwab, "Natural boundary element methods for the electric field integral equation on polyhedra," SIAM J. Numer. Anal., vol. 40 , no. 1

[4] Z. Peng, X. C. Wang, and J. F. Lee, "Integral equation based domain decomposition method for solving electromagnetic wave scattering from non-penetrable objects," IEEE Trans. Antennas Propag., vol. 59, no. 9, pp. 3328-3338, Sep. 2011.

[5] I. Sekulic, E. Ubeda and J. M. Rius, "Nonconforming discretization of the PMCHWT integral equation applied to arbitrarily shaped dielectric objects," European Conference on Antennas and Propagation (EUCAP), Paris, March 2017.

[6] E. Ubeda, Juan M. Rius and A. Heldring, "Nonconforming discretization of the ElectricField Integral Equation for closed perfectly conducting objects," IEEE Transactions on Antennas and Propagation, vol. 62, no. 8, pp. 4171-4186, August 2014. 